\title{
Functional neuroanatomy of the rhinophore of Archidoris pseudoargus
}

\author{
Adrian Wertz $\cdot$ Wolfgang Rössler $\cdot$ Malu Obermayer • \\ Ulf Bickmeyer
}

Received: 4 December 2006 / Revised: 18 January 2007 / Accepted: 18 January 2007 / Published online: 15 March 2007

(C) Springer-Verlag and AWI 2007

\begin{abstract}
For sea slugs, chemosensory information represents an important sensory modality, because optical and acoustical information are limited. In the present study, we focussed on the neuroanatomy of the rhinophores and processing of olfactory stimuli in the rhinophore ganglion of Archidoris pseudoargus, belonging to the order of Nudibranchia in the subclass of Opisthobranchia. Histological techniques, fluorescent markers, and immunohistochemistry were used to analyse neuroanatomical features of the rhinophore. A large ganglion and a prominent central lymphatic channel are surrounded by longitudinal muscles. Many serotonin-immunoreactive (IR) processes were found around the centre and between the ganglion and the highly folded lobes of the rhinophore, but serotonin-IR cell bodies were absent inside the rhinophore. In contrast to the conditions recently found in Aplysia punctata, we found no evidence for the presence of olfactory glomeruli within the rhinophore. Using calcium-imaging techniques with Fura II as a calcium indicator, we found differential calcium responses in various regions within the ganglion to stimulation of the rhinophore with different amino acids. The lack of glomeruli in the rhinophores induces functional questions about processing of chemical information in the rhinophore.
\end{abstract}

Communicated by H.-D. Franke.

A. Wertz · U. Bickmeyer $(\square)$

Biologische Anstalt Helgoland, Alfred Wegener Institut für

Polar- und Meeresforschung, Kurpromenade 201,

27483 Helgoland, Germany

e-mail: Ubickmeyer@awi-bremerhaven.de

\section{A. Wertz $\cdot$ W. Rössler $\cdot$ M. Obermayer}

Department of Behavioral Physiology and Sociobiology,

Biozentrum, University of Würzburg, Am Hubland,

97074 Würzburg, Germany
Keywords Serotonin $\cdot$ Chemosensory $\cdot$ Calcium imaging $\cdot$ Sea slug $\cdot$ Mollusc

\section{Introduction}

Chemical signals play a prominent role in most organisms. Sea slugs, living in shallow waters at the North sea should primarily rely on chemical and mechanical senses, as optical information is limited and the sensitivity of long-range acoustic stimuli without a swim bladder or a sophisticated ear is probably low. Archidoris belongs to the order of nudibranchia, and its rhinophores were shown to be sensitive to mechanical stimulation (Agersborg 1922). Anatomical studies by Storch and Welsch (1969), for the first time, suggested similarities to the osphradium (Welsch and Storch 1969; Wedemeyer and Schild 1995) and that the posterior tentacles, the rhinophores, may serve as chemoreceptive organs. Using electrophysiological recordings and neuroanatomical backfill techniques, Bicker et al. (1982) investigated mechano- and chemoreception in Pleurobranchaea californica. In Aplysia, the rhinophore epithelium was suggested to be chemoreceptive (Audesirk 1975; Emery and Audesirk 1978), and the function of the rhinophore as an olfactory organ was described by Audesirk and Audesirk (1977). Ablation of the rhinophores of Aplysia was shown to cause a decrease in the time spent for mating and the egg laying behaviour, suggesting the sensitivity of the rhinophores for pheromones (Levy et al. 1997; Susswein and Nagle 2004; Cummins et al. 2004). In a recent study using calcium-imaging techniques, Wertz et al. (2006) showed that amino acids are detected and processed by the rhinophores of Aplysia punctata supporting their olfactory function. 
Compared to vertebrates and insects, the olfactory pathway of molluscs has not been investigated deeply. In terrestrial gastropods, the importance of the procerebrum for olfactory information processing was shown (Delaney et al. 1994; Gelperin 1999; Gelperin and Tank, 1990), and the neuroanatomy and function of the cerebrum with respect to olfactory information processing was reviewed by Chase (2000). However, only little is known about the olfactory pathway from the sensory cells to higher centres. In the stylommatophoran terrestric pulmonate Achatina fulica, Chase and Tolloczko (1993) described the anatomy of the posterior tentacle and found olfactory glomeruli with similarities to glomeruli found in vertebrates and arthropods (Hildebrand and Shepherd 1997) and Croll et al. (2003) described glomeruli-like structures in the nudibranch Phestilla. Likewise, the rhinophore of A. punctata possesses a rhinophore ganglion and glomeruli, which are arranged around the rhinophore groove (Wertz et al. 2006). For the genus Archidoris, there is no information available about processing of chemosensory information or the underlying neuroanatomy within the rhinophore. In A. punctata, the rhinophore ganglion and the glomeruli are innervated by centrifugal serotonergic processes (Wertz et al. 2006). Similar to results in the opisthobranchia Pleurobranchaea and Tritonia (Moroz et al. 1997) and sensory organs in Phestilla, serotonergic cell bodies were shown to be absent in the periphery (Croll et al. 2003).

In the present study, we investigated the rhinophore of Archidoris pseudoargus using histological techniques, serotonin immunohistochemistry, and fluorescent tracers to reveal general neuroanatomical features. Responses to amino acids as potential odorants were measured in the rhinophore ganglion using fluorimetric calcium imaging. The results of this study give a first account of the chemosensory structure and function of the rhinophores of A. pseudoargus and open up new avenues for further studies of chemoreception in sea slugs. Differences with recent findings in A. punctata are discussed.

\section{Materials and methods}

Tissue preparation, immunohistochemistry, and fluorescent tracers

Specimens of A. pseudoargus were collected from shallow waters around Helgoland. Animals were of different ages and body sizes. For preparation, the animals were cooled on ice and fixed in $4 \%$ formaldehyde in artificial sea water (ASW; pH 7.5; in mM: $460 \mathrm{NaCl}, 104 \mathrm{KCl}, 55 \mathrm{MgCl}, 11$ $\mathrm{CaCl}_{2}$, and $15 \mathrm{Na}$-HEPES ( $\mathrm{N}$-2-hydroxyethylpiperazine$N$ '-2-ethanesulfonic acid Na-salt). Before further treatments, the rhinophores were washed three times in $0.1 \mathrm{M}$ phosphate buffered saline (PBS, pH 7.2). For labelling with fluophore-conjugated phalloidin, immunohistochemistry, tracing with biocytin markers and nuclear staining, the rhinophores were embedded in 5\% low-melting point agarose (Agarose II, Amresco, Solon, OH, USA, No. 210-815) and sectioned in a frontal or sagittal plane at $150 \mu \mathrm{m}$ with a vibrating microtome (Leica VT 1000S, Wetzlar, Germany). Free-floating agarose sections were preincubated in PBS with $0.2 \%$ Triton $\mathrm{X}-100$ and $2 \%$ normal goat serum (NGS: ICN, Biomedicals, Orsay, France, Cat. No. 191356) for $1 \mathrm{~h}$ at room temperature. Different combinations of double labellings were performed. To label serotonergic neurons, sections were incubated with a primary antibody against serotonin derived from rabbit (1:4000, DiaSorin, Stillwater, MN, USA, Cat. No. 20080, Lot No. 051007) in PBS with $0.2 \%$ Triton X-100 and 2\% NGS overnight at room temperature. This antibody was used successfully in previous studies in gastropod molluscs (Croll et al. 2003; Moroz et al. 1997; Wertz et al. 2006). After five rinses in PBS, sections were incubated in Alexa Fluor 488-conjugated goat anti-rabbit secondary antibody (1:250, Molecular Probes, Eugene, OR, USA, Cat. No. A -11008). To label filamentous (f)-actin in muscles and nervous tissue, sections were incubated in 0.2 units of Alexa Fluor 568 phalloidin (Molecular probes, A-12380) in PBS overnight at $4^{\circ} \mathrm{C}$. To stain cell nuclei, sections were incubated for $15 \mathrm{~min}$ in $25 \mu \mathrm{g} / \mathrm{ml}$ propidium iodide (Molecular probes, P-1304) in PBS with $0.2 \%$ Triton X-100 at room temperature. Sections were finally washed at least five times with PBS, transferred into $60 \%$ glycerol/PBS for $30 \mathrm{~min}$, and mounted on microscopic slides in $80 \%$ glycerol in PBS.

\section{Staining with biocytin}

For live staining with biocytin (Molecular Probes, B1592), rhinophores were transferred to a dish containing ASW, which was then removed by a dry paper. Small amounts of biocytin crystals were applied into the tentacle nerve using a minuten pin. After 2-4 h incubation at room temperature, the rhinophores were fixed in $4 \%$ formaldehyde in ASW for 1 day at $4^{\circ} \mathrm{C}$. After rinsing three times in PBS, rhinophores were embedded in agarose and sliced at $400 \mu \mathrm{m}$ thickness with a vibratome. To visualize biocytin, slices were incubated with streptavidin conjugated with Alexa 488 Fluorophore (1:125, Molecular Probes, S11223) overnight. Following dehydration in an ascending series of ethanol, slices were cleared and mounted in methyl salicylate.

\section{Histology and confocal microscopy}

Rhinophores were fixed in Bouin's fixative solution for 2 days, washed with ethanol, embedded in Spurr's resin, and sectioned in the sagittal and frontal planes $(6 \mu \mathrm{m})$. 
After standard histological procedures, plastic sections were stained on a hotplate after Mallory (1900). Stained sections were washed with distilled water, dried on the hotplate, and mounted in Entellan (Merck, Darmstadt, Germany). Images were taken with a digital Camera (Spotinsight Color, Vistron Systems, Puchheim, Germany) mounted on a microscope (Zeiss Axiophot, Carl Zeiss $\mathrm{GmbH}$, Jena, Germany). Image processing was performed with CorelDRAW (Corel Corporation, Ottawa, Ontario, Canada).

Fluorescent tracers and antibodies were visualized with a laser-scanning confocal microscope (Leica TCS SP) using appropriate lasers, filter settings, and objectives. Image processing was performed with the following software: Zeiss Image Browser (Zeiss GmbH, Jena, Germany), Corel Photopaint and CorelDRAW Graphics Suite (Corel Corporation, Ottawa, Ontario, Canada), and Adobe Photoshop (Adobe, San Jose, USA). Three rhinophores were used for frontal sections and two for sagittal sections applying Mallory stains. Biocytin stainings were perfomed in five rhinophores and all double stainings were replicated at least three times.

Fluorimetric measurements of intracellular $\mathrm{Ca}^{2+}$ levels

The rhinophores were dissected as described above and cut longitudinally using a razor blaze. Sliced rhinophores were incubated for $60 \mathrm{~min}$ at $4^{\circ} \mathrm{C}$ in ASW containing $5 \mu \mathrm{M}$ Fura II acetoxymethylester (AM) (Sigma-Aldrich). After removal of the incubation buffer, the rhinophores were washed for $10 \mathrm{~min}$. Changes in fluorescence were monitored with an imaging system (Visitron, Puchheim) and a CCD camera (Coolsnap cF, Photometrics) mounted on an inverted microscope (Zeiss Axiovert 100) equipped with a UV objective (Zeiss NeoFluar 20×). Different regions within the rhinophore ganglion were measured using the "region" function of the software (Metafluor, Meta Imaging Series, Universal Imaging Corporation). Changes in fluorescence were obtained by ratiometric measurements with excitations at 340 and $380 \mathrm{~nm}$. Values were presented as relative changes in ratios representing alterations in intracellular $\mathrm{Ca}^{2+}$ levels. Fluorescence images were acquired with an interval of $5 \mathrm{~s}$ and an exposure time of $50 \mathrm{~ms}$ per image.

For odour stimulation the recording chamber (volume $3 \mathrm{ml}$ ) was mounted on the microscope stage, and the bath flow was adjusted to $4 \mathrm{ml} / \mathrm{min}$ with a peristaltic pump. The chamber volume was exchanged in less than $1 \mathrm{~min}$. After addition of amino acids to the source beaker the solutions reached the bath chamber after $120 \pm 10 \mathrm{~s}$. Amino acids, which induced the highest responses in $P$. californica (Bicker et al. 1982) and in previous experiments with $A$. punctata (Wertz et al. 2006) were chosen as olfactory stim- uli (Alanine, Arginine, Glutamine, Methionine, and Isoleucine; all purchased at Sigma-Aldrich, Munich, Germany). Amino acids were applied for 1 min at various concentrations with the peristaltic pump system. Each amino acid (1 M stock each) was dissolved in ASW and final concentrations ranged between 2 and $20 \mathrm{mM}$. Stimulus solutions were prepared immediately before use by dissolving the respective stock solution in ASW. After stimulation, ASW was pumped through the recording chamber for at least 10 min to wash out all amino acids. In most cases, 50 regions of interest were measured simultaneously. To test for the viability of the preparations, the last stimulus at the end of an experiment always was a high $\mathrm{K}^{+}$buffer stimulation $(400 \mathrm{mM} \mathrm{NaCl}$ was replaced by $400 \mathrm{mM} \mathrm{KCl})$, which elicited a strong response. Calcium-imaging experiments were performed with $15\left(\mathrm{~K}^{+}\right)$-responsive rhinophores from eight animals using the following application of amino acids. Rhinophore 1: Alanine (Ala), Valine (Val), Histidine (His); 2: Ala, Arginine (Arg), Isoleucine (Ile), Methionine (Met), Glutamine (Gln); 3: Met, Met, Met; 4: Met, Met, Met, Met; 5. Ala, Ala, Arg; 6: Met, His, Val; 7: Ala, Ala, Ala; 7: His, Met, Ala; 8: Met, His, His; 9: Met, Ala, His; 10: Gln, Phenylalanine (Phe), Val, His; 11: Met, Arg, Gln, Gln; 12: Met, Phe, Val; 13: Met, Met, Gln, Ala; 14: repetitive $\left(\mathrm{K}^{+}\right)$; 15: Ala, Met, Ala, Met. Representative experiments are shown in the figures, but not pooled for responses to specific amino acids because of differential responses.

\section{Results}

Neuroanatomy of the rhinophore

For an overview of the general anatomical features of the rhinophore, Mallory-stained histological sections were analysed. Figure 1 shows two sagittal $(a, b)$ and three frontal (c-e) slices of the rhinophore at different planes. The positions of the frontal planes are indicated in Fig. 1a. A large ganglion extends through the longitudinal axis of the rhinophore and multiple side branches of the ganglion project to the rhinophore lobes (Fig. 1f). The neuronal tissue within the ganglion did not show any obvious further compartmentalisation (Fig. 1h), and extends towards the rhinophore lobes (Fig. 1h). Next to the ganglion a prominent lymphatic channel extended through the length axis of the rhinophore with small side branches projecting into the lobes (Fig. 1b, d, g). A perineurial glia-like sheath surrounded the ganglion and its side branches (Fig. 1h, i). The sheath and the ganglion contained cell bodies with a different shape and staining patterns (CB1 and 2 in Fig. 1h, i). Epidermal cells on the rhinophore lobes (Fig. 1g) were covered with cilia, which was supported by phalloidin staining (see below). 
Fig. 1 Histological overview of the rhinophore of Archidoris pseudoargus. a-e: Two sagittal $(\mathbf{a}, \mathbf{b})$ and three frontal (c-e) Mallory-stained sections at different planes of the rhinophore. The locations of the frontal sections are indicated in $\mathbf{a}$. The rhinophore ganglion $(R G)$ extends along the entire rhinophore and has side branches $(S B)$ projecting to the rhinophore lobes $(R L)$. A large central lymphatic channel $(L C)$ is located close to the RG (b-e). In two sagittal sections $\mathbf{f}, \mathbf{g}$ the projection of the side branch $(S B)$ and a bifurcation of the lymphatic channel into the $R L$ is shown. The epidermal cells of the RL possess cilia $(C)$. h, i: Frontal sections of the nervous tissue in the ganglion. Higher magnification of the RG (h) with side branches (i), a glia-like sheath (arrow), different types of cell bodies $(C B 1$ and $C B 2)$ and stained neurofilament $(N F)$. Cross-section of fibres (circle). Scale bar: a-e $=500 \mu \mathrm{m}, \mathbf{f}$, $\mathbf{g}=100 \mu \mathrm{m}, \mathbf{h}, \mathbf{i}=25 \mu \mathrm{m}$
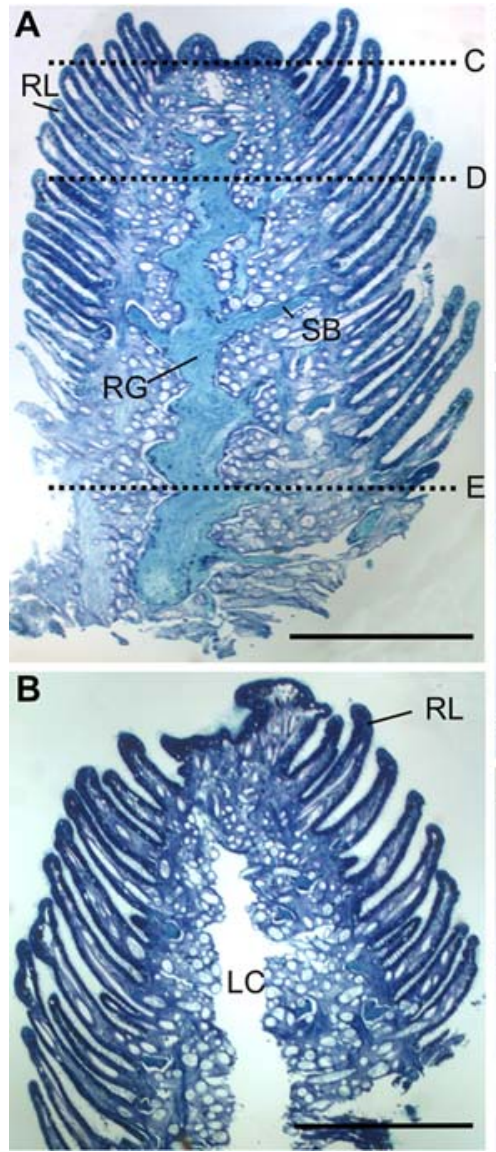

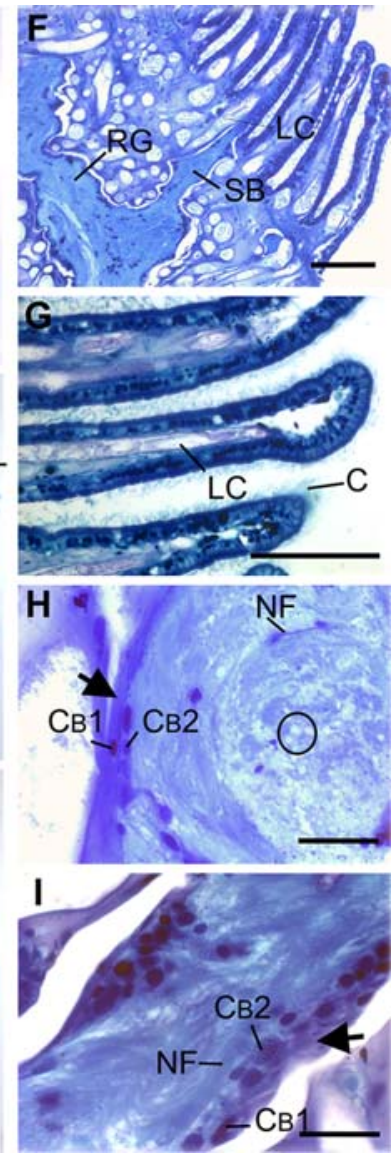

Injection of biocytin in the rhinophore ganglion revealed the neuronal projections into the rhinophore lobes (Fig. 2). Biocytin crystals were applied into the nerve at the base of the rhinophore. The side branches of the ganglion bifurcate and project to the surface of the lobes (Fig. 2). Retrograde staining of cells in the epithelia indicates a direct connection of epidermal cells with the rhinophore ganglion.

Phalloidin labelling revealed prominent bundles of longitudinal muscles (Fig. 3a, b). Interestingly, the entire surface of the rhinophore epithelium was stained with phalloidin. Close inspection indicated that this is most likely caused by cilia on epidermal cells. The rhinophore ganglion was only lightly stained with phalloidin indicating neuronal f-actin within neurons of the rhinophore ganglion.

Figure $3 \mathrm{c}-\mathrm{f}$ shows double stainings with the nucleic acid marker propidium idodide and serotonin-immunoreactivity. Sagittal and frontal slices revealed many cell nuclei in the epithelium as well in and around the rhinophore ganglion. Serotonergic fibres extended from the tentacle ganglion to the lobes in the periphery and were found across the rhinophore. No serotonergic cell bodies were found within the rhinophore. The lymphatic channels (LC) were associated with serotonin-immunoreactivity (Fig. 3f) indicating that these structures are potentially innervated by 5HT IR processes. Most importantly, no glomeruli or glomerulus-like

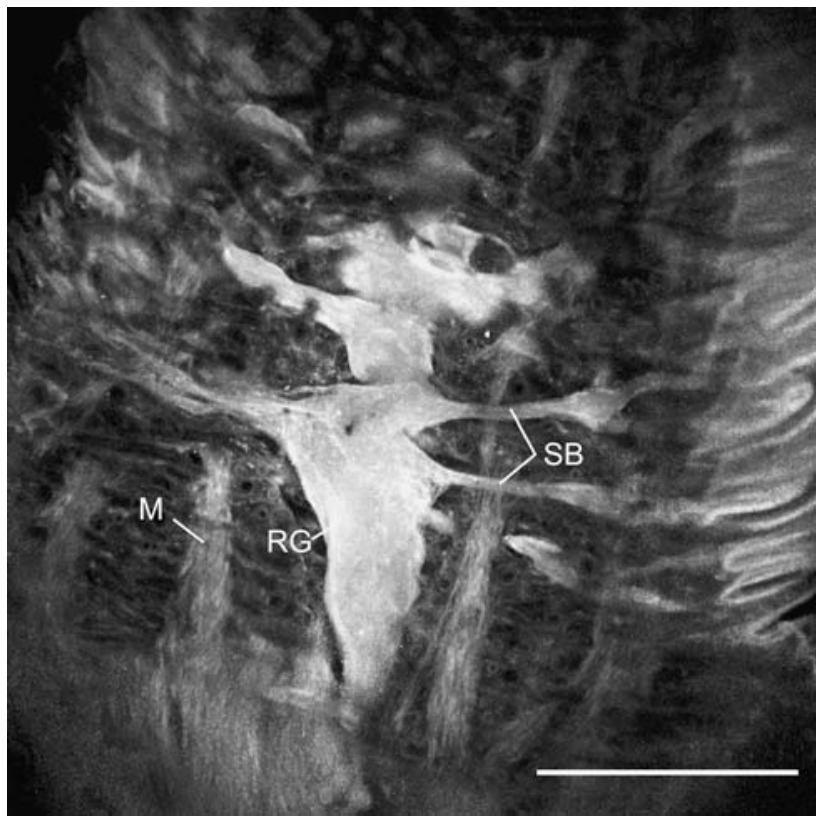

Fig. 2 Biocytin stained processes of the rhinophore ganglion. Sagittal projections of the rhinophore. Biocytin was injected into the tentacle nerve and stained the rhinophore ganglion $(R G)$ and several side branches $(S B)$. The side branches bifurcate and proceed to the periphery into the epithelium of the rhinophore lobes $(R L)$. Scale bar = $500 \mu \mathrm{m}$ 
Fig. 3 Staining with phalloidin, propodium idodide and serotonin-immunoreactivity. Sagittal (a) and frontal view (b) of the rhinophore double labelled with serotonin-immunoreactivity (green) and phalloidin (red). Serotonergic fibres $(S F)$ project from the rhinophore ganglion $(R G)$ to the rhinophore lobes $(R L)$. The epithelial cell layer of the rhinophore lobe $(R L)$ is stained with phalloidin indicating that the cell processes on the surface are cilia $(C)$. Phalloidinlabelled muscle bundles $(M)$ extend only in longitudinal direction. c-f: Double labelling with the serotonin-immunoreactivity (green) and propidium iodide (red). The RG and side branches are surrounded by many cell nuclei $(\mathbf{c}, \mathbf{e})$. Single SF project into the RL (e,f). The small lymphatic channels $(L C)$ are associated with serotonin-immunoreactivity (f, arrow). Scale bars: $\mathbf{a - d}=500 \mu \mathrm{m}, \mathbf{e}, \mathbf{f}=100 \mu \mathrm{m}$
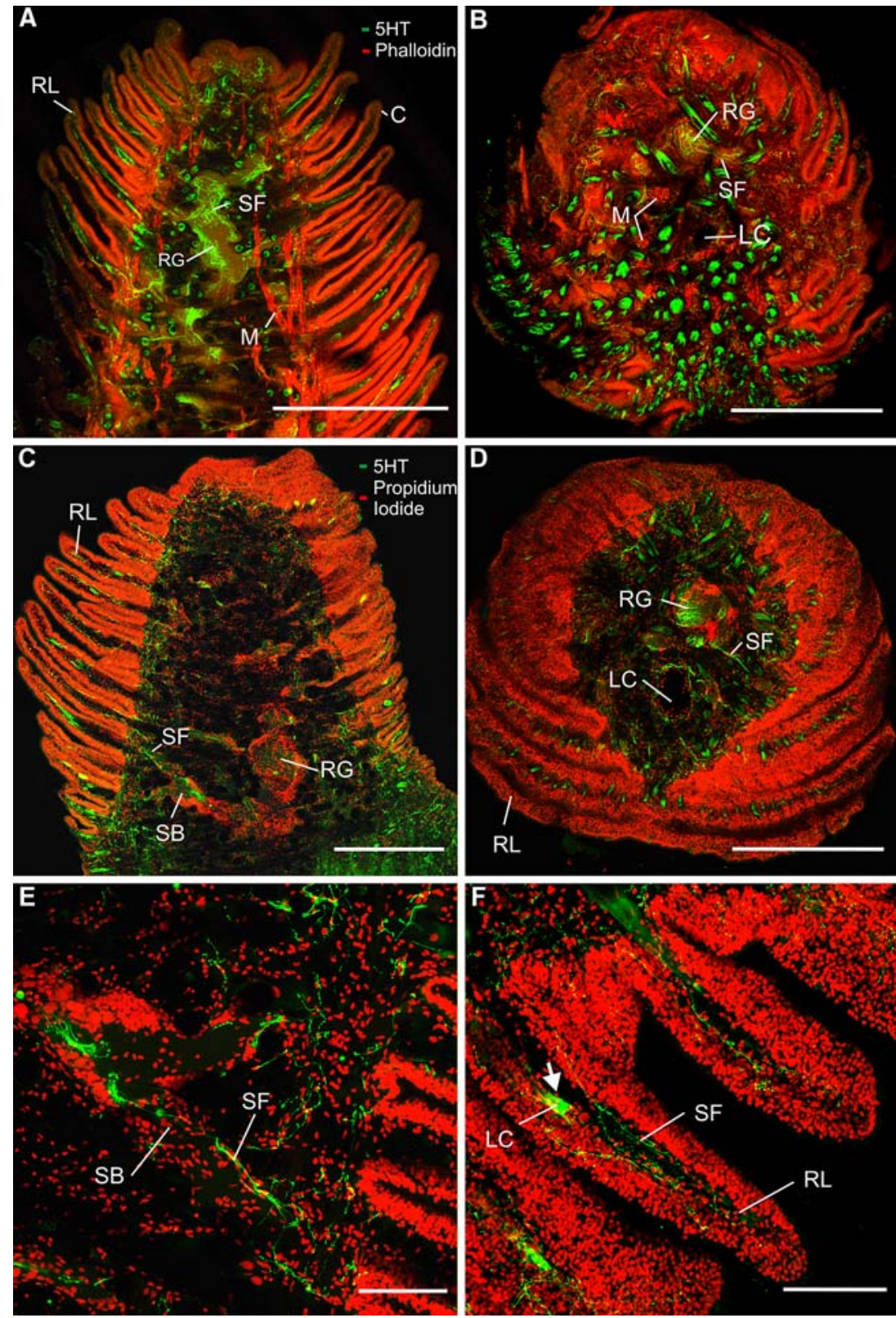

structures were found within the rhinophore or asscociated with the rhinophore ganglion of $A$. pseudoargus.

Calcium imaging of amino acid evoked responses within the tentacle ganglion

Slices of the rhinophore were loaded with Fura II AM and transferred to a calcium imaging setup. The general responsiveness was first tested by application of artificial sea water (ASW) containing high $\mathrm{K}^{+}$. The $\mathrm{Ca}^{2+}$ responses induced by a high $\mathrm{K}^{+}$solution showed a similar increase after repetitive application (result not shown). We chose amino acids as olfactory stimuli, because they were used in previous experiments in gastropod molluscs (Bicker et al. 1982; Murphy and
Hadfield 1997; Wertz et al. 2006). To exclude direct excitation of neurons within the rhinophore, we did not apply glutamic and aspartic acid, which induced highest responses in the nudibranch Phestilla sibogae (Murphy and Hadfield 1997). In 15 experiments we found $\mathrm{Ca}^{2+}$ responses in the rhinophore ganglion induced by the application of the amino acids alanine, arginine, glutamine, methionine, and isoleucine at concentrations of 2 or $20 \mathrm{mM}$. Each amino acid was applied for $1 \mathrm{~min}$ followed by at least $10 \mathrm{~min}$ wash. A schematic drawing indicates the area of measurement of two independent experiments (Fig.4). Within this area different regions of interest were measured for intracellular calcium signals in response to stimulation with amino acids. We omitted all regions which were not responding. 
Fig. 4 Calcium responses within the rhinophore ganglion. a The schematic drawing of the rhinophore illustrates the location of two measurements during two experiments (shown in $\mathbf{b}, \mathbf{d}$ ). The shaded area (in a, c) indicates the measured region $(R G$ : rhinophore ganglion). The position of five regions of interest (ROIs) is shown and signals of these ROIs are plotted in $\mathbf{b}$.

Scale bar $=100 \mu \mathrm{m}$. b: Stimulation with the amino acids (all $20 \mathrm{mM}$ ) alanine (ALA), arginine $(A R G)$, isoleucine (ILE), methionine (MET), glutamine $(G L U)$ and with artifical sea water containing high potassium $\left(\mathrm{ASW}^{\mathrm{K}+}\right)$. Clear responses to ALA and ARG were found in the regions I-IV, whereas ILE, MET, and GLN induced no calcium response. Region $\mathrm{V}$ showed a weak response to ARG. All regions responded to $\mathrm{ASW}^{\mathrm{K}+}$. c: The location of three ROIs of a second experiment is shown in c. d: Signal changes to repeated application of ALA.

Within region I the calcium level decreased, where as the calcium levels of regions II and III increased. e: A simultaneous elevation and decrease in ratio signals measured in different ROIs was found in three additional experiments to the application of ALA, ILE, and GLN
A
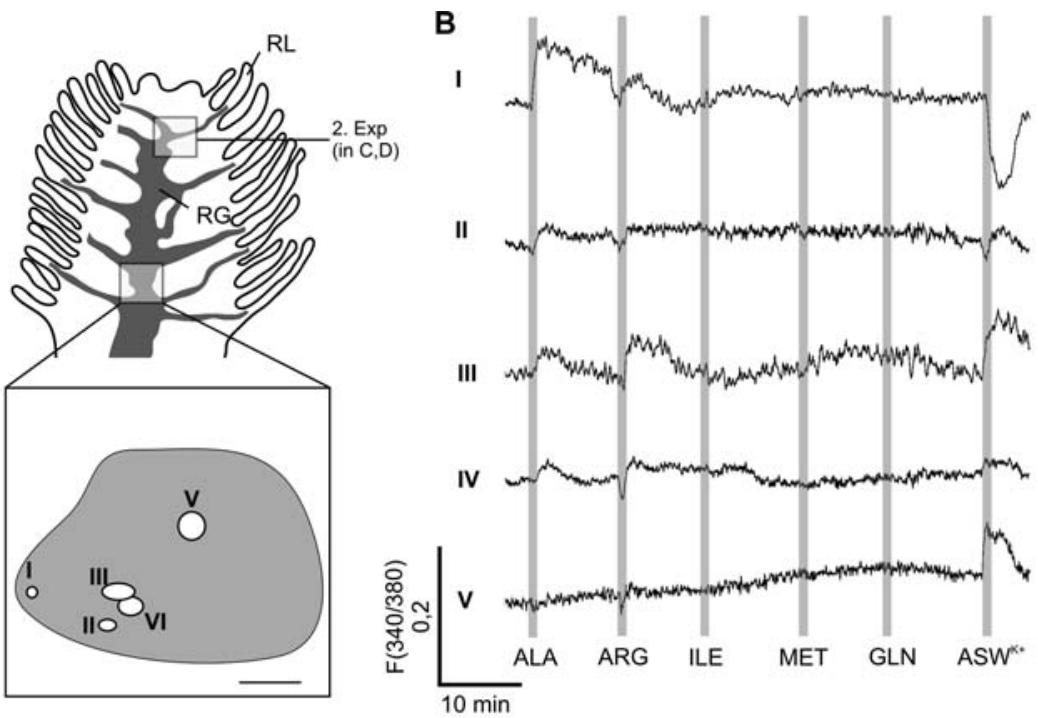

C
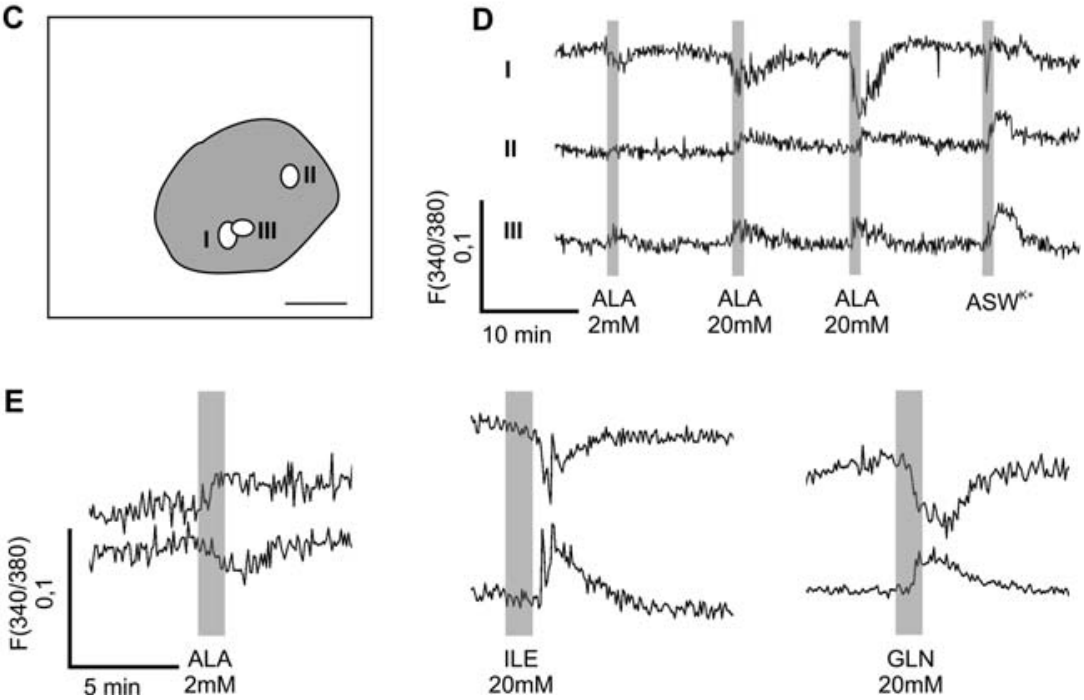
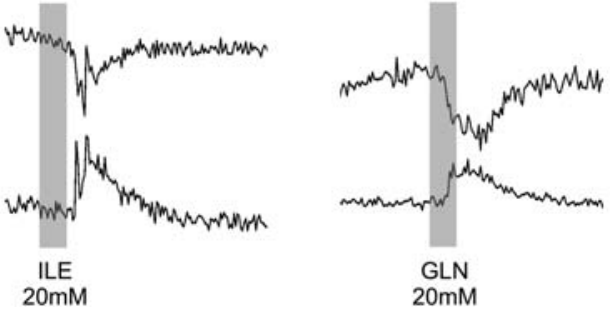

In Fig. 4, five regions are demonstrated which showed responses to the application of amino acids. Strongest responses were recorded to alanine and arginine. All measured regions responded with a change in intracellular calcium levels to arginine, whereas to alanine, region $\mathrm{V}$ did not respond clearly. In this experiment isoleucine, methionine, and glutamine induced no observable changes in intracellular calcium levels. Application of ASW containing high $\mathrm{K}^{+}$induced a calcium level elevation in regions III, $\mathrm{IV}$, and $\mathrm{V}$, whereas region I responded with a calcium level decrease. In region II the calcium level decrease was followed by an increase. With application of arginine such differential responses were observed as well. In the experiment shown in Fig. 4c the same amino acids were applied. The positions of three responding regions are shown. Region I responded with a decrease of intracellular calcium levels, whereas regions II and III showed an elevation. High $\mathrm{K}^{+}$induced a decrease in region I and an increase in II and III. In Fig. 4e, three calcium responses measured in three rhinophores are shown. An increase and a decrease of calcium levels in response to the application of alanine, isoleucine, and glutamine were measured, suggesting complex neuronal processing within the ganglion following stimulation with amino acids.

\section{Discussion}

The rhinophore of A. pseudoargus comprises a prominent rhinophore ganglion. The ganglion is surrounded by a perineurial glial-like sheath. In contrast to the conditions in Aplysia (Wertz et al. 2006) and results obtained in Achatina (Chase and Tolloczko 1993) in histological investigations, serotonin-immunostaining and fluorescent tracing with biocytin did not reveal any olfactory glomeruli inside the rhinophore. In the same line, the homogeneous texture of 
nervous tissue inside the rhinophore ganglion gave no indications of glomerular structures inside the ganglion. Our results are corroborated by new work of the group of Klussmann-Kolb (Faller and Klussmann-Kolb 2006). The findings are in contrast to the presence of glomeruli in most vertebrate and insect primary olfactory centres (e.g. Hildebrand and Shepherd 1997; Rössler et al. 2002). Many serotonergic processes were found to innervate the rhinophore. The origin of these centrifugal serotonergic neurons is not clear, as no serotonergic cell bodies could be determined in the rhinophore, similar to results in other gastropods (Croll et al. 2003; Boudko et al. 1999; Wertz et al. 2006).

The rhinophore contains a lymphatic channel system surrounded by muscular layers, as demonstrated by phalloidin stainings of f-actin. Interestingly the lymphatic channel system appears to be associated with serotonin-IR, which may indicate that the hydraulic system for fast longitudinal movement of the tentacles is under control of serotonergic neurons. Further investigations at the ultrastructural level are needed to resolve the fine structure of potential release sites close to the lymphatic channels. We found no transversal muscles, which otherwise are necessary for expansion of the rhinophores.

Differential alterations (elevation-decrease) of intracellular calcium levels in the presence of single amino acids indicate that chemosensory input is processed in the rhinophore ganglion. The results also demonstrate that various amino acids are detected and differentially processed within the rhinophore ganglion. The lack of any obvious olfactory glomeruli in Archidoris may indicate that the rhinophore may not serve in the first line as an olfactory organ for long distance reception of odorants and may be a primarily tactile or rheotaxic organ. In favour of the rhinophores as an olfactory organ Wyeth and Willows (2006) showed the importance of predator or prey odour plumes for navigational response in Tritonia. Receptor neurons within the rhinophore epithelium of Archidoris may not be very sensitive to stimulation with amino acids. Responses to tactile stimuli and other chemical substances such as alkaloids used in Aplysia (Bickmeyer et al. 2004) need to be investigated in the future to clarify this issue.

Stimulation with alanine, isoleucine, and glutamine induced calcium elevations as well as decreases in the ganglion possibly reflecting inhibitory and excitatory influences and neuronal processing of chemosensory information in the ganglion. Another possibility could be direct inhibition and excitation of sensory cells by chemical stimuli, similar to results found in crustaceans (Michel et al. 1991) and squids (Danaceau and Lucero 1998; Lucero et al. 1992). Further studies of the sensory neurons within the rhinophore epithelium are needed to clarify these aspects of sensory reception and processing of chemical stimuli in Archidoris.
In conclusion, a large ganglion and a prominent central lymphatic channel surrounded by longitudinal muscles are present in the rhinophore. Many serotonin-immunoreactive (IR) processes but no serotonin-IR cell bodies were found inside the rhinophore. No evidence for the presence of olfactory glomeruli within the rhinophore can be presented. Different amino acids are detected by the rhinophore.

Acknowledgments We thank the Helgoland diving group, in particular Carsten Wanke, Saskia Brandt and the late Udo Schilling for collecting Archidoris.

\section{References}

Agersborg HPK (1922) Some observations on qualitative chemical and physical stimulations in nudibranchiate mollusks with special reference to the role of the 'rhinophores'. J Exp Zool 36:423-445

Audesirk TE (1975) Chemoreception in Aplysia californica. I. Behavioral localization of distance chemoreceptors used in food-finding. Behav Biol 15:45-55

Audesirk TE, Audesirk GJ (1977) Chemoreception in Aplysia californica. 2. Electrophysiological evidence for detection of odor of conspecifics. Comp Biochem Physiol A 56:267-270

Bicker G, Davis WJ, Matera EM (1982). Chemoreception and mechanoreception in the gastropod mollusk Pleurobranchaea californica. 2. Neuroanatomical and intracellular analysis of afferent pathways. J Comp Physiol 149:235-250

Bicker G, Davis WJ, Matera EM, Kovac MP, Stormogipson DJ (1982) Chemoreception and mechanoreception in the gastropod mollusk Pleurobranchaea californica. 1. Extracellular analysis of afferent pathways. J Comp Physiol 149:221-234

Bickmeyer U, Drechsler C, Köck M, Assmann M (2004) Brominated pyrrole alkaloids from marine Agelas sponges reduce depolarization-induced cellular calcium elevation. Toxicon 44:45-51

Boudko DY, Switzer-Dunlap M, Hadfield MG (1999) Cellular and subcellular structure of anterior sensory pathways in Phestilla sibogae (Gastropoda, Nudibranchia). J Comp Neurol 403:39_ 52

Chase R (2000) Structure and function in the cerebral ganglion. Microsc Res Techn 49:511-520

Chase R, Tolloczko B (1993) Tracing neural pathways in snail olfaction-from the tip of the tentacles to the brain and beyond. Microsc Res Techn 24:214-230

Croll RP, Boudko DY, Pires A, Hadfield MG (2003) Transmitter contents of cells and fibers in the cephalic sensory organs of the gastropod mollusc Phestilla sibogae. Cell Tiss Res 314:437-448

Cummins SF, Nichols AE, Rajarathnam K, Nagle GT (2004) A conserved heptapeptide sequence in the waterborne attractin pheromone stimulates mate attraction in Aplysia. Peptides 25:185-189

Danaceau JP, Lucero MT (1998) Betaine activates a hyperpolarizing chloride conductance in squid olfactory receptor neurons. Journal of Comparative Physiology A-Sensory Neural and Behavioral Physiology 183:225-235

Delaney KR, Gelperin A, Fee MS, Flores JA, Gervais R, Tank DW, Kleinfeld D (1994) Waves and stimulus-modulated dynamics in an oscillating olfactory network. Proc Natl Acad Sci U S A 91:669-673

Emery DG, Audesirk TE (1978) Sensory cells in Aplysia. J Neurobiol 9:173-179

Faller S, Klussmann-Kolb A (2006) Transmitter distribution in the cephalic sensory organs of Ophistobranchia. In: 2nd international ophistobranch workshop (Bonn) Abstract p 21 
Gelperin A (1999) Oscillatory dynamics and information processing in olfactory systems. J Exp Biol 202:1855-1864

Gelperin A, Tank DW (1990) Odor-modulated collective network oscillations of olfactory interneurons in a terrestrial mollusk. Nature 345:437-440

Hildebrand JG, Shepherd GM (1997) Mechanisms of olfactory discrimination: converging evidence for common principles across phyla. Annu Rev Neurosci 20:595-631

Levy M, Blumberg S, Susswein AJ (1997) The rhinophores sense pheromones regulating multiple behaviors in Aplysia fasciata. Neurosci Lett 225:113-116

Lucero MT, Horrigan FT, Gilly WF (1992) Electrical responses to chemical-stimulation of squid olfactory receptor-cells. J Exp Biol 162:231-249

Mallory FB (1900) A contribution to staining methods: I. A differential stain for connective-tissue fibrillae and reticulum. II. Chloride of iron haematoxylin for nuclei and fibrin. III. Phosphotungstic acid haematoxylin for neuroglia fibres. J Exp Med 15-20

Michel WC, Mcclintock TS, Ache BW (1991) Inhibition of lobster olfactory receptor-cells by an odor-activated potassium conductance. J Neurophysiol 65:446-453

Moroz LL, Sudlow LC, Jing J, Gillette R (1997) Serotonin-immunoreactivity in peripheral tissues of the opisthobranch molluscs
Pleurobranchaea californica and Tritonia diomedea. J Comp Neurol 382:176-188

Murphy BF, Hadfield MG (1997) Chemoreception in the nudibranch gastropod Phestilla sibogae. Comp Biochem Physiol A 118:727735

Rössler W, Kuduz J, Schurmann FW, Schild D (2002) Aggregation of F-actin in olfactory glomeruli: a common feature of glomeruli across phyla. Chem Senses 27:803-810

Storch V, Welsch U (1969) Cytology and function of the nudibranch rhinophores. Z Zellforsch 97:528-536

Susswein AJ, Nagle GT (2004) Peptide and protein pheromones in molluscs. Peptides 25:1523-1530

Wedemeyer H, Schild D (1995) Chemosensitivity of the osphradium of the pond snail Lymnaea stagnalis. J Exp Biol 198:1743-1754

Welsch U, Storch V (1969) Osphradium of prosobranch gastropods Buccinum undatum and Neptunea antiqua. Z Zellforsch 95:317330

Wertz A, Rössler W, Obermayer M, Bickmeyer U (2006) Functional neuroanatomy of the rhinophore of Aplysia punctata. Front Zool $3: 6$

Wyeth RC, Willows AO (2006) Odours detected by rhinophores mediate orientation to flow in the nudibranch mollusc, Tritonia diomedea. J Exp Biol 209:1441-1453 